\title{
SPATIOTEMPORAL ENVIRONMENTAL HEALTH MODELLING: \\ A Tractatus Stochasticus
}




\title{
SPATIOTEMPORAL ENVIRONMENTAL HEALTH MODELLING: A Tractatus Stochasticus
}

\author{
by \\ George Christakos \\ and \\ Dionissios T. Hristopulos \\ School of Public Health \\ Department of Environmental Sciences and Engineering \\ The University of North Carolina at Chapel Hill
}

Springer Science+Business Media, LLC 


\section{Library of Congress Cataloging-in-Publication Data}

\section{Christakos, George.}

Spatiotemporal environmental health modelling : a tractatus stochasticus / by George Christakos and Dionissios T. Hristopulos.

p. $\mathrm{cm}$.

Includes bibliographical references and index.

ISBN 978-1-4419-5048-2 ISBN 978-1-4757-2811-8 (eBook)

DOI 10.1007/978-1-4757-2811-8

1. Environmental health--Mathematical models. 2. Stochastic processes. I. Hristopulos, Dionissios T. II. Title.

RA566.C58 1998

616.9'8'015118--dc21

Copyright (C) Springer Science+Business Media New York 1998

Originally published by Kluwer Academic Publishers in 1998.

Softcover reprint of the hardcover 1st edition 1998

All rights reserved. No part of this publication may be reproduced, stored in a retrieval system or transmitted in any form or by any means, mechanical, photocopying, recording, or otherwise, without the prior written permission of the publisher, Springer Science+Business Media, LLC 
Dedicated to the memory of my father Panayiotis, who gave and cared so much,

and to my mother Mary who continues to do so.

\section{George Christakos}

To my parents, Theodoros and Anna, and my wife, Lisa, for their love and support;

To Greece, for all that she has given me.

Dionissios Hristopulos 


\section{Forward}

"La pensée n'est qu'un éclair au milieu de la nuit. Mais c'est cet éclair qui est tout"."

H. Poincaré

Protecting and promoting health at the population level requires a rigorous interdisciplinary scientific base. Health is the product of an array of complex physiological, environmental, and behavioral processes. Success in understanding and addressing these processes require the capacities of many different scientific disciplines. The science of public health has made remarkable advances in recent years as its practitioners have explored new ways of linking and applying knowledge from core disciplines in the mathematical, natural, and behavioral sciences. Environmental health is an essential element of public health science, and its concepts and methods epitomize the interdisciplinary nature of public health.

The successful integration of concepts and methods from diverse fields of study requires both creativity and depth of knowledge. At its best, such integration creates a scientific synergism that enables new insight regarding the problems and issues under study. In this book, George Christakos and Dionissios Hristopulos create just such a synergism for the practice of environmental health science. The authors explore a new and powerful approach to environmental health science --one which brings together principles from modern stochastic theory with more traditional methods of examining environmental processes and their associate health effects. This approach offers fresh views of common environmental health issues, and new insight regarding methods for assessment and control.

Public health scientists, decision-makers, and students alike have much to gain from the concepts and methods described in this book. By advancing our understanding of the natural and physiological processes which determine health, the authors make a substantial contribution to the field of public health and its mission of assuring population health. In doing so, the authors underscore the importance of interdisciplinary scientific approaches to public health issues.

William L. Roper, MD, MPH

Dean, School of Public Health

The University of North Carolina at Chapel Hill 


\section{Preface}

"We haven't the money, so we've got to think."

E. Rutherford

This book is the culmination of an investigation into the applicability of modern stochastics to problems of environmental health science. The result is a stochastic interpretation of processes (physical, chemical, biological, epidemic, etc.) that account for changes in the state of the environment and human health. This interpretation exposes the inherent uncertainty in such processes, and advocates prediction in terms of spatiotemporal probability laws and statistical statements. The deterministic approach is based on the hypothesis that a complete characterization of the system is available. Deterministic theories provide inadequate representations for most environmental health processes, due to the incomplete knowledge of the underlying mechanisms and the partial characterization of model parameters. In the stochastic viewpoint the focus is shifted from the analysis of a single, perfectly determined system, to an ensemble of possible systems, which represent all the potentialities permitted by the existing knowledge of the system's properties. Thus, instead of concentrating on single system properties, the stochastic approach calculates ensemble properties. Finally, conclusions about the former are derived on the basis of the latter. Stochastic analysis can be viewed as a mathematical generalization of conventional deterministic analysis. This generalization is more flexible in practice and can handle problems in environmental health science more accurately and efficiently than the ofteninadequate deterministic methods. In recent years a new synthesis has been emerging in the study of natural phenomena that combines physical models with stochastic methods, which account for uncertainties and variabilities. The new synthetic view, called modern stochastics, and its applications in environmental health science will be the focus of this book. The answers that modern stochastics supplies are not always obvious to the deterministically minded, and in certain cases deep-rooted prejudices may need adjustment.

As its title dictates, this book uses stochastic concepts and methods to build links between models and techniques of environmental sciences, on the one hand, and health sciences on the other. To our knowledge, this is a task that is not shared by any other book currently available in the literature. It is, however, a natural development, because the environment and human health are closely related and interdependent in many ways. 
Rigorous quantitative analysis of the associations between, e.g., subsurface contaminant transport models and human exposure, or between space-time ozone distribution and disease incidence rates, are of great significance in health management. Such associations are crucial for site selection and human exposure assessment in epidemiologic studies. Another example is carcinogenesis, which is a random multistep process controlled both by cancer genes and environmental conditions. Separated, environmental and health models rarely lend each other assistance; only when their associations and interactions have been clearly established, they can illuminate and enrich one another.

In light of the above considerations, one may distinguish two main objectives of the book. The first is to provide a coherent and unified account of modern spatiotemporal stochastics for the environmental health scientist. At this level the book can function as a textbook as well as a reference volume, since it contains a number of novel methods and results in stochastic environmental health modelling. The second goal is to apply modern stochastics in environmental health science problems in a way that emphasizes their close relations and strong interdependence. Of course, since environmental health science is progressing very fast and in various directions, no text of any reasonable size can exhaust all the possibilities. Therefore, in this book we have chosen to focus on a number of carefully selected applications. However, modern stochastics has applications in phenomena that cover a considerably wider range than what is presented here, and practically includes every aspect of environmental health science.

Although this is definitely an application-oriented book, it contains several philosophical theses and comments on methodological issues. The reason is that, in our view, modern stochastics is not just a collection of abstract equations and techniques for problem solving. Instead, it constitutes a scientific paradigm based on a more flexible conceptualization of reality than its deterministic counterpart. Efficient use of the stochastic paradigm presupposes an appropriate conceptual framework, within which the significance and emerging applications of notions such as uncertainty, variability, heterogeneity and risk can be debated in the scientific forums. In addition, the new paradigm can help scientists develop an ability to argue coherently and convincingly on a wide range of issues, a skill which is extremely important in communicating their efforts to broader audiences in the public and political arenas.

Throughout the book our approach is conceptual, having a strong pedagogic orientation with many examples presented for edification and assimilation of the conceptual principles and the mathematical techniques. The variety of applications also provides a framework for evaluation, and a realization that there can be a systematic organization for many concepts, tools and perspectives. Furthermore, the aim of this approach has been to find quantitative 
solutions to environmental health problems that are serviceable to research scientists and practitioners who work in these fields.

Based on the fact that the roots of many useful stochastic concepts and tools have multiple branches that penetrate different scientific disciplines, the book has been written with a diverse audience in mind. It should be of interest to scholars and researchers in the environmental sciences, epidemiology, health sciences, statistics, risk analysis, mathematics, and decision making. Furthermore, practitioners in the above areas will find a variety of theoretical and computational tools that should be of immediate practical use. The book is also intended as a textbook for graduate-level modelling courses in environmental modelling, health risk assessment, geostatistics, descriptive epidemiology, biostatistics, and medical geography.

In writing this book our approach has been guided by the following dictum: "True education is what survives when a person has forgotten all that he/she has been taught." The aim of this book goes beyond providing a compendium of technical recipes, black-box computer algorithms and simplified remedies for certain problems. Instead, this book aims to give aspiring scientists some motivation and tools for addressing environmental health issues with a critical mind. The book should be read in the same spirit, and we encourage the reader to question the arguments that we use, and to think of counter-examples. The book is intended to stimulate thought, not to be an alternative to it. If readers examine the material presented critically, they will discover areas where they may agree or disagree with the authors. It is our hope that in either case they will clarify their own beliefs and, perhaps, prejudices in the process.

We are grateful to several colleagues for stimulating discussions, especially to C.T. Miller, D. Crawford-Brown, P. Bogaert and G. Cassiani. Valuable contributions were made by the first author's former and current students, V.M. Vyas, B.R. Killam, J-J. Lai, X. Li, D.L. Oliver, G. Thesing, A. Kolovos, M. Serre and K-M. Choi. Finally, we greatfully acknowledge funding from the National Institute of Environmental Health Sciences (Grant no. P42 ES05948-02), the Department of Energy (Grant no. DE-FC09-93SR18262), and the Army Research Office (Grant no. DAAL03-92-G-0111). Without their support, this book would have never been written, for better or for worse.

George Christakos

Dionissios Hristopulos

Chapel Hill, N.C. 
Note: The following notation is used throughout the book:

- $\quad \$ 5$ denotes the 5 th section in the same Chapter.

- $\quad \$ I I .3$ denotes the 3 rd section of Chapter II.

- $\quad$ Eq. (4.2) denotes the 2 nd equation of section 4 in the same Chapter.

- $\quad$ Eq. (IV.9.5) denotes the 5th equation of section 9 in Chapter IV. This notation is used when we refer to an equation from within a different Chapter. 


\section{Table of Contents}

Chapter I: FUNDAMENTAL PRINCIPLES OF STOCHASTIC ENVIRONMENTAL HEALTH MODELLING

1. On the method of environmental health science. 1

2. Fundamental principles of the stochastic mode of thinking. 7

3. The notion of spatiotemporal continuity. 16

3.1 Philosophical aspects of space and time 17

3.2 The space-time continuum concept 18

$\begin{array}{ll}3.3 & \text { Natural vs. health processes } \\ 3.4 & \text { Space-tim }\end{array}$

3.4 Space-time coordinates 20

4. The field concept in environmental health science 22

5. Knowledge bases in the space-time domain. 26

6. Spatiotemporal scales. 27

6.1 Spatiotemporal scales for natural processes 28

6.2 Spatiotemporal scales for health processes 31

6.3 The hierarchy of scales 32

7. An overview of the environmental health paradigm 34

Chapter II: ENVIRONMENTAL EXPOSURE FIELDS

AND THEIR HEALTH EFFECTS 37

1. The holistic environmental exposure -health effect perspective 37

2. Studying environmental exposure fields 42

2.1 Pollution sources 42

2.2 Environmental pollutants 43

2.3 Characterization of the environment 44

2.4 Environmental compartments 45

2.5 Environmental media 46

$\begin{array}{lll}2.6 & \text { Natural fields } & 47\end{array}$

2.7 Evolution mechanisms 49

3. Human Exposure Concepts 53

3.1 Basic notions 53

3.2 Mathematical expressions for exposure 59

3.3 Mathematical expressions for biomarkers 64 
xiv

3.4 Exposure as a practical biomarker estimator $\quad 68$

4. Health Effect Concepts 69

4.1 The health impact pathway and the various kinds of health effects 69

4.2 Mathematical models for health effect indicators 71

4.3 Mathematical models for individual-based and population-based dose and $\begin{array}{ll}\text { exposure-response curves } & 73\end{array}$

$\begin{array}{lll}4.4 & \text { Spatiotemporal coordinates in human exposure analysis } & 77\end{array}$

Chapter III: SPATIOTEMPORAL RANDOM FIELDS IN EXPOSURE ANALYSIS AND ASSESSMENT

1. A theatrical introduction to the random field concept 81

2. The spatiotemporal random field 83

2.1 Basic mathematical formulation 84

2.2 Characterization in terms of ensemble functions 87

3. S/TRF classifications 90

4. Space-time metrics 95

5. Spatiotemporal correlation models 97

$\begin{array}{lll}5.1 & \text { General properties } & 97\end{array}$

5.2 Criteria of permissibility 99

5.3 Useful tools for constructing spatiotemporal correlation functions 101

6. Separable space-time covariance models 102

6.1 Separability in the space-time domain 102

$\begin{array}{ll}6.2 \text { Classes of spatial covariance models } & 103\end{array}$

7. Natural models and covariance functions 109

8. Regression S/TRF models 114

$\begin{array}{lll}8.1 & \text { Mathematical formulation } & 114\end{array}$

$\begin{array}{ll}\text { 8.2 Statistical inference issues } & 116\end{array}$

9. Wave representation of S/TRF 118

Chapter IV: $\quad$ MODELLING EXPOSURE HETEROGENEITIES 121

1. A class of spatiotemporal random fields 121

$\begin{array}{lll}1.1 & \text { The central idea and definitions } & 121\end{array}$

1.2 Determination of the $Q$-operator in practice and its physical significance 125

$\begin{array}{lll}1.3 & \text { An S/TRF decomposition } & 127\end{array}$ 
2. Spatiotemporal polynomial notation 127

3. Orders of spatiotemporal continuity $v / \mu \quad 129$

4. Continuum representations of S/TRF- $v / \mu \quad 132$

5. Discrete representations: spatiotemporal increments of orders $\nu / \mu 135$

6. Spectral Representations 139

7. Generalized spatiotemporal random fields 141

$\begin{array}{ll}\text { 8. Permissibility criteria } & 147\end{array}$

8.1 Analysis in the continuous spatiotemporal domain -

The generalized spatiotemporal covariance 148

8.2 Some examples in the discrete space-time domain 152

$\begin{array}{lll}\text { 8.3 Permissibility conditions for polynomial covariances } & 154\end{array}$

$\begin{array}{lll}8.4 & \text { Permissibility conditions for vector S/TRF } & 157\end{array}$

9. Generalized covariance models derived from PDE's 160

10. Space-time separability 163

11. New generalized spatiotemporal covariance models 164

$\begin{array}{ll}11.1 & \text { The Polynomial model } \\ 11.2 & 164\end{array}$

$\begin{array}{ll}11.2 & \text { The Exponential model } \\ 11.3 & 165\end{array}$

$\begin{array}{ll}11.3 \text { The Gaussian model } & 169\end{array}$

Chapter V: SPATIOTEMPORAL MAPPING OF ENVIRONMENTAL HEALTH PROCESSES - THE BME APPROACH 171

1. About spatiotemporal maps 171

2. Space-time mapping fundamentals 173

2.1 The basic epistemic framework of the BME approach 173

$\begin{array}{ll}2.2 & \text { Other mapping approaches } \\ 2.3 & 175\end{array}$

$\begin{array}{lll}2.3 & \text { Operational concepts of scale } & 176\end{array}$

3. Mathematical formulation of the BME approach 177

4. Some analytical results 185

$\begin{array}{lll}4.1 & \text { Multi-point BME } & 185\end{array}$

$\begin{array}{ll}4.2 & \text { Single-point BME } \\ & 186\end{array}$

5. Other formulations of the BME approach 189

$\begin{array}{lll}5.1 & \text { Functional BME } & 189\end{array}$

$\begin{array}{lll}5.2 & \text { Vector BME } & 191\end{array}$

6. BME vs. MMSE estimates 192 
$\mathrm{xVi}$

Chapter VI: SPATIOTEMPORAL MMSE MAPPING

1. Introduction 193

2. Mathematical Formulation 194

3. Linear MMSE space-time mapping 195

$\begin{array}{lll}3.1 & \text { Mathematical formulation } & 195\end{array}$

$\begin{array}{lll}3.2 & \text { Recursive formulations } & 199\end{array}$

$\begin{array}{lll}3.3 & \text { More properties } & 199\end{array}$

4. Homogeneous-Stationary S/TRF: Space-time Kriging forms 201

4.1 Biased MMSE mapping 201

4.2 Unbiased MMSE mapping 202

5. Nonhomogeneous-nonstationary S/TRF: The case of S/TRF- $v / \mu 206$

6. Vector MMSE space-time mapping 212

7. Implementation issues - Applications 214

$\begin{array}{lll}7.1 & \text { Practical map generation } & 214\end{array}$

$\begin{array}{lll}7.2 & \text { Trend determination } & 221\end{array}$

8. Regression S/TRF 224

9. BME vs. MMSE estimators 226

10. BME vs. Kriging estimators 226

Chapter VII: STOCHASTIC PARTIAL DIFFERENTIAL EQUATION

MODELLING OF FLOW AND TRANSPORT 231

1. Introduction 231

2. Perturbation expansions 235

3. The diagrammatic approach 240

3.1 The diagrammatic language - Physical space 241

3.2 Diagrams in frequency space 243

4. Self-consistent equations $\quad 247$

$\begin{array}{lll}4.1 & \text { The basic concepts } & 247\end{array}$

4.2 The asymptotic limit 251

5. Diagrammatic methods in subsurface hydrology 252

6. Renormalization group analysis-Change of scale 253

6.1 The basic concepts 254

6.2 Diagrammatic approximations for the self-energy 258

6.3 Frequency space RNG treatment of transport 259 
6.4 Perturbative RNG schemes 261

6.5 RNG analysis of subsurface pollution transport 263

7. The space transformation technique 265

7.1 Basic definitions 265

7.2 Some properties of STs 267

7.3 Numerical implementations of ST 270

7.4 Applications of STs in SPDE solving 272

7.5 Differential geometric approach to multiphase flow 275

7.6 Boundary effects 276

Chapter VIII: STOCHASTIC PHYSIOLOGICALLY-BASED

POLLUTOKINETIC MODELLING 279

1. Introduction 279

2. Compartmental analysis of pollutokinetics 280

3. One-compartment stochastic pollutokinetics 284

4. Multi-compartment stochastic pollutokinetics 290

5. Stochastic pollutokinetics and health effects 292

6. Some generalizations 297

Chapter IX: STOCHASTIC EXPOSURE AND HEALTH INDICATORS 299

1. Introduction 299

2. Stochastic exposure indicators 302

2.1 The exposure S/TRF-pair 303

2.2 The stochastic exposure indicator concept 305

2.3 One-point stochastic exposure indicators 306

2.4 Two-point stochastic exposure indicators 309

2.5 Uncertainty in the exposure threshold level 313

2.6 Other properties of exposure indicators 315

2.7 Ergodicity and sample averaging of exposure indicators 318

3. Stochastic cell-based health effect indicators 322

3.1 Cellular structures 322

3.2 The threshold postulate 323

3.3 Relationships between the basic cell indicators 329

3.4 Derived cell indicators 331 
Xviii

3.5 Scale and modelling effects 331

3.6 The multistage postulate 335

4. Stochastic formulation of traditional population

health effect indicators $\quad 340$

4.1 Two fundamental health effect indicators 341

4.2 Person-time rates 346

4.3 Rate ratios 349

4.4 Spatiotemporal variation and mapping of population health indicators The case of the North Carolina breast cancer incidence distribution $\quad 349$

5. Correlation analysis 355

5.1 Population health damage indicators 355

5.2 Linear PER model 359

5.3 Uniform population density 360

5.4 Stochastic trends and uncertainty assessment in exposure-damage models 361

5.5 Time delayed PER relationships 363

6. Spatiotemporal maps of health damage indicators - The ozone case 363

7. Analysis of disease - Exposure association 369

$\begin{array}{ll}\text { Bibliography } & 375\end{array}$

$\begin{array}{ll}\text { Index } & 391\end{array}$ 